\title{
Das Jenseits: ein Traum - die Translation: ein Spiel
}

\author{
Interkulturelle Funktionsstellen \\ in Rhonabwys Traum (Breuddwyd Rhonabwy)
}

MatThias DëUmer

\begin{abstract}
In order to participate in intercultural discourse, one way for medieval literary criticism could be to work on intersections between historical intercultural exchanges and the way these exchanges are reflected in the today's image of medieval times and literature. This article works on the example of adoptions and exclusions of foreign cultural elements in the Welsh text Breuddwyd Rhonabwy (Rhonabwy's Dream). In the multilayered structure of this medieval text, >dream < and >game< work as systems of intercultural exchange. The final question is: Can these systems nowadays still be functionalized to convey the otherness of medieval Welsh culture?
\end{abstract}

Title: The Beyond: a Dream - The Translation: a Game. Intercultural Functions in Rhonabwy's Dream (Breuddwyd Rhonabwy)

Keywords: medieval culture; welsh culture; Arthurian literature; dream; game

\section{Methodische VorüberLegung}

Als 1991 in Straßburg der II. Internationale Kongress der Gesellschaft für Interkulturelle Germanistik abgehalten wurde, stand die Großtagung ideologisch im Zeichen der deutschen Wende und dem Fall des Eisernen Vorhangs. Überfliegt man das Inhaltsverzeichnis des über tausend Seiten umfassenden Bandes, der aus diesem Kongress erwuchs, so fällt auf, dass unter den beinahe 90 kulturund literaturwissenschaftlichen Beiträgen sich nur drei mediävistische (oder zumindest am Image des Mittelalters interessierte) finden lassen (vgl. Batts 1993; Shitanada 1993; Müller 1993). Diese quantitativ geringe Beteiligung der Mediävistik an der Entwicklung interkultureller Ansätze ist in den frühen 1990er Jahren kein Einzelfall: Auch im zeitnah erschienenen und von Peter Zimmermann herausgegebenen Band zur Interkulturellen Germanistik (vgl. Zimmermann 1991) herrscht zwar ein Interesse daran, Deutsch als Fremdsprache zu methodisieren (und DAF als Fach zu institutionalisieren), aber eben als gegenwärtige Fremdsprache, teilweise sogar unter gezieltem Ausschluss sprach-historischer Alteritäten. Die Beweggründe für diese Exklusion sind so offensichtlich wie verständlich: Die Ausrichtung der Interkulturellen Germanistik in ihrer (Re-)Etablierungs- 
phase war gegenwartsbezogen und synchron ausgerichtet; man reagierte auf das akute Bedürfnis, die kulturellen und sprachlichen Vermittlungsprobleme nach dem Fall von Mauer und Vorhang zu lösen. So blieb zu wenig Spielraum für den diachronen Blick auf das Mittelalter.

Dabei ist Ulrich Müller unbedingt recht zu geben, dass die Vermittlung des Fremden zu den Grundlagen der mediävistischen Literaturwissenschaft gehört, in der schulischen oder universitären Lehre, in der kulturellen wie sprachlichen Übersetzungsarbeit, aber letztendlich auch in allgemeineren gesellschaftlichen Zusammenhängen und ideologischen Prozessen. Angesichts literarischer Rezeptionen mittelalterlicher Stoffe in zeitgenössischen Werken kommt Müller zu der Aussage: »All dies lenkt den Blick auf etwas nur scheinbar Paradoxes: Die für die Germanistik derzeit wohl interessantesten interkulturellen Probleme sind nicht in der exotischen Fremde, aus einer extremen Fremd-Perspektive zu beobachten, sondern in der allernächsten Nähe, nämlich innerhalb Deutschlands.« (Müller 1993: 855)

Müllers Plädoyer für eine engagierte Mediävistik kann mittlerweile nicht mehr auf die Rezeptionsforschung und die Zeit der Wende beschränkt werden. Gegenwärtig sind die integrativen Anforderungen an die europäische Gemeinschaft (unter anderem, aber nicht ausschließlich durch Geflüchtete) tendenziell noch größer - der Standpunkt der Mediävistik scheint sich hingegen kaum geändert zu haben. Davon, dass der (mögliche) Beitrag der Mediävistik zur interkulturellen Theoriebildung noch immer zu den marginalisierten Positionierungen gehört, zeugt Mathias Herwegs Aufsatz in der letztjährigen ZiG. Herwegs Anliegen ist es, »den ästhetischen und didaktischen Wert des schon sprachlich Fremden inmitten der eigenen literarischen Kultur auszuloten.« (Herweg 2017: 12) Dazu zieht er sich nicht wie Müller auf die Rezeptionsforschung zurück, sondern stellt vielmehr den gegenwärtigen Erkenntnisgewinn dar, der durch eine Überkreuzung diachroner und synchroner Interkulturalität im Umgang mit den mittelhochdeutschen Texten entstehen kann: »Das Nibelungenlied oder der Parzival können uns Heutigen Leseerfahrungen über etwas vermitteln, das die Gegenwartsliteratur allenfalls in der sog. Migrationsliteratur bereithält.« (Ebd.: 13) Herweg macht deutlich, dass die über das Alteritätsparadigma argumentierende diachrone Interkulturalität zwar ideologisch den affirmativen Aneignungen eines >deutschen< Mittelalters (das es niemals gegeben hat) entgegensteht, jedoch zugleich in »einer unter den Aufklärern grassierenden Mittelalterverachtung « (ebd.: 18) wurzelt. Dabei scheint es letztendlich gleich, ob das Mittelalter sich eine abgrenzende Funktionalisierung zum >dunklen Mittelalter< oder aber den nicht weniger dunklen affirmativen Aufwertungen im Rahmen der Nationenbildung im 19. Jahrhundert oder im deutschen Faschismus gefallen lassen muss, denn »Alterität und Kontinuität sind zwei Seiten der gleichen Medaille.« (Ebd.: 14)

Diesem ideologischen Oszillieren diachroner, am Mittelalter ausgerichteter Interkulturalität zwischen Aneignung und Alterität stehen diverse synchrone Austauschprozesse des Mittelalters zur Seite, die jedoch anders gelagert sind als die Beschreibung von interkulturellen Austauschvorgängen neueren Datums - 
und, so möchte ich meinen: besser gelagert, wenn es darum geht, der Falle der nationalistischen Aufladung zu entgehen. Wie Reinhold Görling gezeigt hat, ist $>$ Kultur $<$ durchaus ein »Hot Thing «, von dem man manchmal besser die Finger lässt (Görling 1999): Zu nah ist der Begriff noch immer verwoben mit dem der >Nation<. Dies jedoch ist für den Kulturbegriff des Mittelalters anders. Es gibt, oder eher (angesichts der auch noch gegenwärtigen wissenschaftssprachlichen Norm, die recht unbedarft die Begriffe >deutsch < und >deutschsprachig< gleichsetzt): Es sollte keine Blaupause der Nation geben, vor der Kultur beschrieben werden kann. Die Grundlagen der kulturellen Unterschiede sind im Mittelalter andere: Zwar sind sie sprachlich, jedoch keinesfalls nationalistisch, häufig religiöser, ideengeschichtlicher und oft auch ganz banal geographischer, doch nur selten geopolitischer Natur. Eine interkulturelle Mediävistik muss diese Prä-Nationalität des Mittelalters als ideologischen Vorteil zu nutzen verstehen. ${ }^{1}$

Eine der Mediävistik eigene Methodik der Interkulturalität, die als engagiertes Fach auch gegenwartsbezogen wirken will, wäre also als Kreuzung von diachronen und synchronen Ansätzen jenseits der Nationsfiktion zu beschreiben. Dann könnte das Nachzeichnen historischer Vermittlungen zwischen Fremdem und Eigenem im Mittelalter zugleich bedeuten, auch den eigenen, zeitgenössischen Umgang mit dem Fremden neu zu denken. Das Ziel wäre somit die Beschreibung von Intersektionen, in denen die Fremdheit im Mittelalter der Fremdheit des Mittelalters begegnet, genauso wie das Prä-Nationale dem AntiNationalen heutiger Ideologien.

\section{SYNCHRONE INTERKULTURALITÄT ZWISCHEN INSELKELTISCHEN Arthurmythen Und kontinentalen Artusromanen}

Generell muss man eine solche Methode erst einmal in ihren historiographischen Möglichkeiten einschränken. Der Verlauf von interkulturellen Interferenzen ist für das Mittelalter eine oft nur schwer beweisbare Sache. Man muss von mündlichen Übermittlungen ausgehen, die über lange Strecken und religiöse wie sprachliche Grenzen hinweg stattfanden. Nur im Sonderfall führten diese zu textuellen Zeugnissen; in diesen kann man maximal nach >Spuren<, also nach dem erstarrten Nachleben solcher Vermittlungsprozesse, suchen.

Für das Wechselspiel zwischen den französischsprachigen Artusromanen und den mittelkymrischen Arthurmythen ist die Ausgangslage jedoch verhältnismäßig ergiebig. Das liegt vor allem daran, dass sich an den französischsprachigen Höfen eine Schriftkultur entwickelte, die auf den Umgang der Inselkelten $^{2}$ mit ihren eigenen Sagenstoffen rückwirken konnte. Man hat es also mit

1 I Siehe hierzu auch den Beitrag von Claudia Brinker-von der Heyde im vorliegenden Band.

2 | Bei der Verwendung von 'keltisch oder ,Kelten a als Bezeichnung einer Kultur ist die Heterogenität dieses Begriffs mitzudenken, die durch eine historische Gemengelage, aber vor allem auch durch differierende disziplinäre Verwendungen des Begriffs be- 
einem Ineinander von Interkulturalität und Intermedialität zu tun. Aufgrund der (auch) medialen Strahlkraft der französischsprachigen Artusromane konnte Helmut Birkhan die erhaltenen mittelkymrischen Arthurmythen als Verschriftlichungen von autochthonen Sagen und den Texten identifizieren, die auf Basis des Chrétien'schen Werks entstanden sind (vgl. Birkhan 1989: 27-44).

Rhonabwys Traum ist wahrscheinlich am Ende des 12. Jahrhunderts entstanden und eventuell im 13. Jahrhundert fertiggestellt worden (vgl. ebd.: 201f.). Die Erzählung stellt einen generischen Zwitter dar. Ohne Frage reagiert Rhonabwys Traum auf den kontinentalen Artusroman; im Gegensatz zu den anderen Überlieferungen erzählt er jedoch keinen Chrétien'schen Plot nach, sondern stellt das Autochthone der inselkeltischen Sagenwelt als Eigenes, aber auch teilweise Überlebtes aus. Es sind somit zwei synchron-interkulturelle Austauschverläufe zu bedenken, an deren Ende dieser Text steht: einerseits die Verarbeitung walisischen Erzählguts durch Chrétien, als dieser den kontinentalen Roman entwickelte; andererseits die Translation der kontinentalen Romane ins Kymrische, die sich wiederum auf das kultureigene Erzählen der Waliser auswirkte.

\subsection{Die Ebenenschachtelung von Rhonabwys Traum}

Obwohl Rhonabwys Traum vor allem aufgrund seiner äußert farbigen und traumhaft-fluiden Bildlichkeit wohl als der kryptischste Text gelten kann, den die postChrétien'sche kymrische Literatur zu bieten hat, wartet er mit einer sehr stringenten Binnenontologie auf. Der Plot besteht aus (mindestens) drei ineinander verschachtelten Ebenen. Die äußerste ist die Erzählung von einem historischen Bruderstreit zwischen Maredudd und Iorwerth, der im 12. Jahrhundert im Königreich Powys stattfand und zur jüngsten Vergangenheit der Textentstehung zu rechnen ist (vgl. ebd.). Im Rahmen dieses Konflikts wird Rhonabwy von Maredudd ausgesendet, um den rebellierenden Bruder Iorwerth zu finden. Auf dieser Suche kommt Rhonabwy mit seinen Männern in einer Hütte unter. Als Anführer des Suchtrupps übernachtet er auf einem vom Text auffällig betonten gelben Kalbsfell, das, nachdem er einschläft, wie ein Traumportal funktioniert.

Die zweite Ebene ist dann der titelgebende Traum. Rhonabwy begegnet darin Iddawg, mit Beinamen >Der Aufwiegler von Britannien<. Geleitet von diesem besucht er Arthur und dessen Heer kurz vor dem Eintritt in die Schlacht von Camlaan, also jenem Ereignis, mit dem die arthurische Herrschaft enden wird. Rhonabwys Führer hat seinen Beinamen daher, dass er im Vorfeld dieser Schlacht Arthur als Bote diente, dieses Amt jedoch ausnutzte, um den Konflikt zwischen Arthur und seinem Neffen Medrawt zuzuspitzen. Für diese Intrige, so lässt der >Aufwiegler< es Rhonabwy wissen, habe er bereits gebüßt.

dingt ist (vgl. Niehues 2013: 217). Ich meine im Kommenden den walisischen, mittelkymrisch schreibenden Anteil der Inselkelten, den ich (die heuristische Setzung ist mir bewusst) als kulturelles System behandle, das mit dem (ebenso heuristisch gesetzten) System der französischsprachigen kontinentalen Höfe im Austausch stand. 
Die dritte Ebene entsteht durch die Schwelle zwischen (Traum-)Realität und Spiel. Diese ist ebenfalls durch eine Unterlage örtlich markiert: Vor den Augen des träumenden Protagonisten wird Arthurs Unsichtbarkeitsmantel auf einer Wiese ausgebreitet. So, wie Rhonabwy auf dem gelben Kalbsfell nächtigt und träumt, setzten sich nun Arthur und Owein in diesen eingezirkelten Ort, um mehrere Partien Gwyddbwyll (übersetzt etwa »Holz-Sinn«) zu spielen, ein walisisches Brettspiel. ${ }^{3}$

Abbildung 1: Die Binnenontologie von Rhonabwys Traum (eigene Darstellung)

\section{REZEPTIONSEBENE}

Text - (Rezitator) - Rezipient

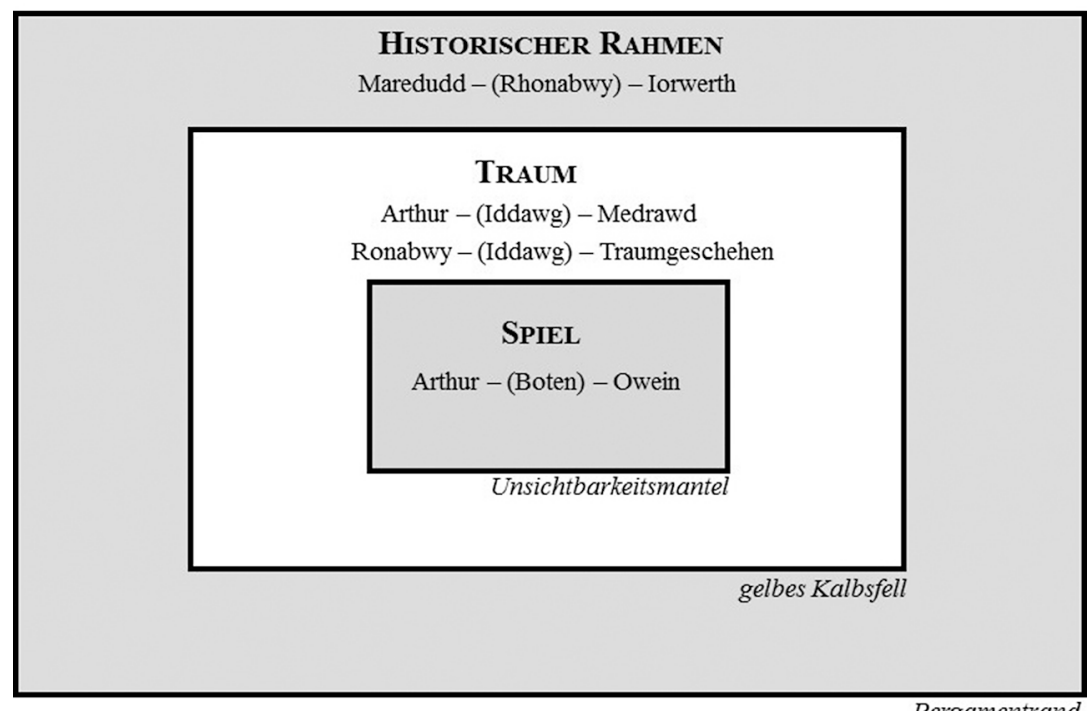

Pergamentrand

Wenn man die physischen Umrandungen, das Kalbsfell für die Traum- und den Unsichtbarkeitsmantel für die Spielsphäre, in ihrer Logik fortsetzt, lässt sich als äußerste Schwelle die Textgrenze nennen, also - materiell und im medialen Modus der Skripturalität dargestellt - der Rand des Pergaments. Dabei

3 | Häufig wurde dieses Spiel mit dem Schach gleichgesetzt, teilweise auch als solches übersetzt. Wie zuletzt Jan Niehues nachgewiesen hat, ist dies eine Fehleinschätzung. Zwar handelt es sich um ein "Kampfspiel", das "in der Regel vom jeweils besseren Spieler gewonnen wird" (im Gegensatz zu Jagd- oder Rennspielen), jedoch sind die Regeln nicht auf die des Schachs zurückzuführen. Gwyddbwyll ist Teil einer autochthonen Spielkultur, die sich von anderen schon dadurch unterscheidet, dass den hölzernen Spielsteinen ein eigenes Bewusstsein, eben ein 'Sinn zugeschrieben wurde, was das Vorkommen sich selbst spielender Spielsteine in mehreren Texten erklären könnte. Aufgrund von datierbaren Lautveränderungen der keltischen Sprachen ergibt sich für das Spiel als terminus ante quem das 6. Jahrhundert (vgl. Niehues 2013: 225-233, hier 230). 
scheint zumindest das Material der beiden äußeren Grenzen metonymisch aufeinander bezogen, ist doch Pergament nichts anderes als die Haut unter dem (gelben) Kalbsfell. Der Unsichtbarkeitsmantel wiederum vermag antithetisch auf das Kalbsfell bzw. dessen ungewöhnliche Farbe zu verweisen, wird er doch »[d]er Weiße« genannt, weil er »keine andere Farbe auf sich als die eigene [duldet]« (Rhonabwys Traum 1989: 130).

Diese auffällige Verkettung der physischen Schwellenmarker hat zweierlei zur Folge. Einerseits wird daraus die Analogie von Text, Traum und Spiel deutlich, eine Entsprechung, die sich als mehrfach gestaffelte Markierung von Uneigentlichkeit begreifen lässt: So wie der Traum nicht das eigentliche historische Geschehen ist, so ist auch das Spiel eine gesellschaftliche Konvention, welche die in ihren Grenzen stattfindenden Handlungen als uneigentliche ausweist. Der vom Pergament getragene Text wäre dann die Ebene, von der aus alle weiteren (im Sinn eines proto-fiktionalen Denkens) uneigentlich sind. ${ }^{4}$

\subsection{Das Spiel ums Autochthone}

Das Spiel, das Arthur und Owein betreiben, ist eines, das direkte Einflüsse auf sie umgebende kriegerische Handlungen nimmt: Die Spielzüge haben stets Entsprechungen in einem Kampf von Arthurs Gefolgsleuten gegen Oweins RabenKrieger, von welchem Boten den beiden Spielern berichten. Interessanterweise sind es nicht die Ereignisse der Schlacht von Camlaan, die durch die Spielzüge beeinflusst werden, sondern ein davon unabhängiger Kampf, was als Zeichen dafür gelten kann, dass die konventionellen Grenzen des Spiels zu der gleichen Systemschließung führen wie die Grenzen des Traums.

Arthurs Gegner Owein hat eine eigene historische sowie Sagentradition, sodass in der Spielkonkurrenz - laut Birkhan - »deutliche Reflexe der ehemaligen sagenmäßigen Rivalität zwischen beiden Heldenkönigen « (Birkhan 1989: 22) zutage treten würden. Davon zeuge auch, dass Oweins Krieger die >Raben< sind. Von diesen nimmt man an, dass sie aus einer autochthonen walisischen Geschichte stammen, die Owein mit Rabenvögeln assoziierte (vgl. Breulmann 2009: 75). Diese Vermutung rührt daher, dass die >Raben < am Ende der Geschichte der Gräfin vom Brunnen (Owein or chewedyl iarlles y ffynnawn) (vgl. Birkhan 1989) als die Gefolgschaft genannt werden, zu der Owein nach Erfüllung des Aventiurewegs zurückkehrt.

Doch dieser Aventiureweg ist gar nicht Oweins eigener, denn in der Gräfin vom Brunnen wandelt er unabhängig vom Autochthonen auf den Spuren von Chrétiens Yvain. Owein ist mit Blick auf diesen Text der walisische Held, der seine autochthone Identität im Rahmen der Beeinflussung durch die kontinentalen Artusromane am stärksten aufgeben musste. Die >Raben < unterliegen der gleichen Ambivalenz, denn einerseits könnten sie auf Autochthones verweisen,

4 | Als unterste wäre auch noch die vom Spielbrett eingefasste Ebene der Spielfiguren zu denken. Da diese aber keine Beschreibung oder Alice in Wonderland-artige Belebung erfährt, ist sie rein hypothetisch. 
andererseits findet man sie gerade am Ende jener Dichtung, die am stärksten kontinental geprägt ist..$^{5}$ Die Wiederaufnahme der Raben in Rhonabwys Traum könnte also entgegen Birkhans Lesart auch bedeuten, dass hier der Owein gemeint ist, der zum Wiedergänger des kontinentalen Yvain reduziert wurde. Mit dieser Lesart treten in Arthur und Owein nicht zwei >eigene< Heldenkönige gegeneinander an, sondern vielmehr Allegorien der beiden Stufen mittelkymrischer Dichtung, die sich durch das (im Gwyddbwyll symbolisierte) Wechsel>Spiel< von Eigenem und Fremdem ergeben.

Arthur wird so zum Inbegriff eines Erzählens, das durch die Figurenidentität eine Kontinuität prä- und post-Chrétien'scher Erzählungen erreicht. Owein hingegen, der sein Image als Heldenkönig ablegen musste, um zum Protagonisten einer wiedererzählten kontinentalen Dichtung zu werden, steht für die Aspekte des arthurischen Erzählens, die der keltischen Kultur von den kontinentalen Romanen aus der Hand genommen wurden. Diese narrativen Stränge kontrastiert Rhonabwys Traum miteinander und macht somit die innerste Textebene zu einem Sinnbild der zeitgenössischen interkulturellen Austauschbewegungen: der Einfluss des walisischen Arthurs auf den kontinentalen Roman einerseits, die Rückwirkung dieses Romans auf die walisische Erzählkultur andererseits.

Im Gwyddbwyll siegt der neue, kulturfremde narrative Strang. Entscheidender jedoch ist, dass Arthur, Inbegriff des Urtümlichen, mit einer Vernichtung des verbindenden Systems >Spiel< (bzw. der Literatur) reagiert, indem er wütend das Spielbrett umstößt. Da in dieser Szene die Allegorien nicht nur um literarische Vormacht, sondern auch um eine kulturelle Identität spielen, die sich in dieser Literatur spiegelt, muss man die Szene recht kulturpessimistisch deuten: Zwar kann das kontinentale Erzählen (Owein) im Spiel (also innerhalb der literarischen Regeln) die Oberhand behalten; Arthur jedoch (also das walisisch Eigene) hat die Macht, dieses gesamte System zu zerstören und den Austauschprozess damit zu beenden.

\subsection{Der traumdeutende Aufwiegler}

Die zweite interkulturell-literarische Beeinflussung betrifft Iddawg, den Aufwiegler von Britannien, der im Traum Rhonabwy leitet. Er scheint in seiner Rolle als >Traumführer< stark beeinflusst durch die Gestalt des Angelus Interpres, wie ihn die Textreihe der Jenseitsreisen kennt. ${ }^{6}$ Nicht nur arbeitet der mittelkymrische Text mit vergleichbaren Frage/Antwort-Formeln, wie sie die Gespräche zwischen Reisendem und Engel in den Jenseitsreisen prägen (vgl. Benz 2013); ;

5 | Noch stärker sprechen die Raben für eine 'kontinentale Gefolgschaft‘, wenn man sie - dies halte ich aber für fraglich - funktional mit Yvains Bündnis mit dem Löwen gleichsetzt (vgl. Breulmann 2009: 75).

6 | Allgemein zu Jenseitsreisen, Visionsliteratur und dem Status des Angelus Interpres vgl. Däumer 2016b.

7 | In Rhonabwys Traum kann man bei der ersten Begegnung der Figuren (vgl. Rhonabwys Traum 1989: 123) die Fragen des Protagonisten noch als Teil einer herkömmli- 
nicht nur treten diese Formeln in Kombination mit einer Variation der $>$ Und ich/er sah ...- - und siehe<-Formulierungen auf, die in den Jenseitsreisen darauf ausgelegt sind, die Wahrnehmung des Reisenden mit der der Rezipierenden zu verschalten (vgl. Däumer 2015 und 2016a); ${ }^{8}$ auch für die Absicherung der Wahrnehmung, die in den Jenseitsreisen häufig gefährdet ist, wird gesorgt: materialisiert in Form des Steins an Arthurs Ring, der auf magische Weise bewirkt, dass Rhonabwy sich an alles erinnern wird, nachdem er aus dem Traum erwacht ist. ${ }^{9}$

Iddawgs Vorgeschichte wird ebenfalls vor der Folie der Angelus-InterpresFunktion in den hochmittelalterlichen Jenseitsreisen, welche im Wesentlichen Conversio-Narrative sind (vgl. Weitbrecht 2011), im besonderen Maße sinntragend: Im Gegensatz zum erklärenden Engel ist Iddawg kein Medium eines göttlichen Wissens, vielmehr führt ihn seine Vorgeschichte als verderbtes Medium ein, das seine Vermittlerposition zwischen Arthur und Medrawd missbrauchte. Durch seine Rolle als verlässlicher Traumdeuter für Rhonabwy tut er dafür Buße. Wie bei den hochmittelalterlichen Jenseitsreisen ist also ein Conversio-Narrativ vorhanden; jedoch ist dieses auf den >Engel<, nicht auf den Reisenden bezogen.

Gestalten, die durch das Unbekannte und Unverständliche leiten, sind in der mittelalterlichen Literatur zahlreich vorhanden. Es lassen sich in Rhonabwys Traum aber auch tiefer gehende, strukturelle Übereinstimmungen mit den Jenseitsreisen finden. Zuerst natürlich ist es Iddawgs Buße, die einen an das Fegefeuer denken lässt, das ab dem 12. Jahrhundert in den Jenseitskonzepten Popularität gewinnen konnte (vgl. Le Goff 1990). Diese Purgatoriumsartigkeit des Traums führt zu Paradoxien, die ebenfalls in den Jenseitsreisen geprägt sind. So kopiert der walisische Autor beispielsweise die typische Zeitparadoxie, die in den Jenseitsreisen durch die Kombination der Eigenzeit des Reisenden und dem Konzept eines allzeitigen Jenseits entsteht (vgl. Däumer 2016b): Einerseits be-

chen Dialogführung betrachten; danach jedoch läuft alles Weitere nach dem Jenseitsreisen-Muster 'Wahrnehmung - Frage - erläuternde Antwort ab (vgl. ebd.: 124-128). Mit der Spielpassage zwischen Arthur und Owein wechselt die Fokalisierung, sodass sich die Formeln nicht mehr finden lassen. Danach jedoch setzt die Handlung wieder mit einer Frage ein, die aufgrund des Ebenenwechsels in indirekter Rede formuliert werden muss, bevor die gewohnte Formel wieder einsetzt (vgl. ebd.: 139f.).

8 | In Rhonabwys Traum ist das meist eine Verschaltung einer primär akustischen Wahrnehmung mit dem imaginativen Blick des Rezipierenden (unter anderem: "[U]nd als sie in die Richtung des Lärms schauten, siehe da! « Rhonabwys Traum 1989: 124). Mit Einsatz des Spiels beziehen sich die Wahrnehmungsapostrophen auf eben dieses; nach dem Spiel setzen sie wieder mit Rhonabwy/lddawg als focalizer ein (vgl. ebd.: 139).

9 | An dieser Stelle ist auffällig, dass die Wahrnehmungsversicherung über eine Verkehrung der Frage/Antwort-Formel erfolgt, sodass der Engel der Fragende ist: "Und dann sagte Iddawg: , Rhonabwy, siehst du an der Hand des Kaisers den Ring und den Stein darin?، Jar, antwortete er. 'Einer der Wunderkräfte des Steines ist es, daß du dich an das erinnern wirst, was du heute nacht sahst. Hättest du den Stein aber nicht gesehen, so würdest du nichts von den Abenteuern behalten.' (Ebd.: 125) 
sichtigt Rhonabwy Arthurs Heer vor seinem Aufbruch nach Camlaan; andererseits ist dem Aufwiegler nach einer siebenjährigen Buße bereits vergeben worden (vgl. Rhonabwys Traum 1989: 127). Iddawg besucht mit Rhonabwy also seine eigene Vergangenheit und einen Arthur, der eigentlich schon seit mindestens sieben Jahren tot sein müsste.

Über die Kombination der Zeitparadoxie mit dem Conversio-Motiv wird die narrative Leitlinie der hochmittelalterlichen Jenseitsreisen (Sünde - Reise - Bekehrung - Verbreitung des Erfahrenen) einer Binnenparadoxie unterworfen: Dem Sünder wurde schon vor den katastrophalen Folgen seiner Tat vergeben. In einem Vorgang der interkulturellen Translation wurde der jenseitige Raum für das walisische Publikum zur mythischen Traumebene und die Kausalität von Sünde und Vergebung zum zeitlosen und damit >gleich-gültigen Z Zustand.

Die Medialität wird jedoch nicht nur im Boten und Traumdeuter Iddawg thematisiert, sie durchzieht vielmehr alle Ebenen des Texts: Auf der Spielebene sind es die Boten, die Arthur und Owein von den Toden berichten, die ihre Spielzüge jenseits des Unsichtbarkeitsmantels verantworten; auf Traumebene ist es die besagte Doppelfunktion von Iddawg und auf Ebene der historischen Rahmung ist es die Botenfunktion, die Rhonabwy selbst innehat: Er soll, wie die Rahmenerzählung, die nie zu Ende geführt wird, beschreibt (vgl. ebd.: 119f.), zur Suche nach dem feindlichen Iorwerth ausziehen. Zum Schluss erfährt man lediglich, dass Rhonabwy auf dem gelben Kalbsfell erwacht und drei Tage und drei Nächte geschlafen hat (vgl. ebd.: 142). Dann bricht der Plot ab und der Text endet in einem Epilog ohne Handlungsrelevanz (vgl. ebd.).

Die ausbleibende Schließung der historischen Rahmenhandlung ist nicht von fragmentarischem Charakter, sondern ein konzeptionell offen angelegtes Ende. Alle Ebenen verweisen über Boten auf ihr Äußeres: Arthurs und Oweins Spiel korrespondiert mit Kampfeshandlungen, von denen Boten ihnen berichten; die Bilder des Traums beeinflussen Rhonabwy, weil Iddawg sie erläutert. In Erweiterung dieser Verweise stellt der Text es (nicht zuletzt über Arthurs magischen Memorial-Ring) an den Erwartungshorizont seines offenen Endes, dass der erwachende Protagonist nun nicht mehr als Häscher, sondern als vermittelnder Bote die Reise fortsetzen wird.

\section{DiE SYNCHRON-DIACHRONE INTERSEKTION von SPIEl Und Traum}

Es gibt in Rhonabwys Traum also zwei Systeme, in denen Fremdes mit dem eigenen Erzählen vermittelt wird: Auf Spielebene ist es der Vermittlungsvorgang mit der kontinentalen Prägung Oweins, auf Traumebene ist es der Einbezug der Jenseitskonzeption. Man könnte in ihrer Funktion das Spiel und den Traum als literarische Simulation dessen beschreiben, was Homi K. Bhabha als >Dritte Räume < bezeichnet, als Zonen eines Zusammentreffens von Kulturen, die eine Wandlung derselben ermöglichen, indem gegenseitige Beeinflussungen angenommen, verändert oder abgewiesen werden (vgl. Bhabha 1994). Ermöglicht 
werden die Aushandlungen, da in diesen Dritten Räumen das ursprüngliche Machtgefälle aufgelöst wird, es also weder eine wahrnehmende Primärkultur noch eine imperialistisch dominierende gibt. Diese Funktionen sind in Rhonabwys Traum sowohl auf der Traum- wie auf der Spielebene vorhanden: Einerseits gewinnt die kontinentale Kultur (symbolisiert durch Owein) das Spiel, andererseits weiß Arthur (als Symbol des Autochthonen) dieses Spiel im Gesamten zu beenden. Für die Traumebene gilt dies genauso: Einerseits wird das Erzählen vom Jenseits in seinen Mechanismen übernommen, andererseits wird es statt auf Glaubens- auf eine Traumebene versetzt.

Wenn diese Ebenen aber im 12./13. Jahrhundert solcherart simuliert werden, um interkulturelle Prozesse auszuhandeln, sollte es, als Einlösung der methodologischen Ankündigung dieses Beitrags, möglich sein, eine Überkreuzung dieser historischen Interkulturalität mit einer diachronen aufzuzeigen.

Für das Spiel kann dies gelingen. Die Marburger Keltologen Franziska Bock und Jan Niehues haben bei einer Tagung zu >Spielen<, die im Juli 2017 in Berlin stattfand, zeigen können, wie wichtig das Marktsegment der Strategie- und Brettspiele dafür ist, ein Bild (oder vielmehr Klischee) der keltischen Kultur in unserer heutigen Gesellschaft $\mathrm{zu}$ entwickeln. ${ }^{10}$ Gerade das 2011 erschienene Spiel Song of Arthur and Merlin geht mit einer bemerkenswerten Bedachtsamkeit die interkulturelle Vermittlung an. Beispielsweise stellt die Einleitung die Frage: »Which King Arthur to play?«, und bietet den Spielenden die Möglichkeit, entweder den kontinental-literarischen »High Medieval King «, den mythischen »Welsh King« oder aber den »Historical King Arthur« zu spielen (vgl. Mersey 2011: 3). Je nach Entscheidung sind die beiliegenden Figuren anders zu bemalen und die Regeln zu variieren. Diese Unterscheidung zwischen dem hochmittelalterlich-literarischen, dem walisisch-mythischen und dem historischen Arthur kann als Veranschaulichung dafür dienen, wie reflektiert eine interkulturelle, dritträumliche Vermittlung (einheitlich imaginierter) keltischer Kultur über das Spiel angesteuert werden kann. Diese moderne Vermittlung des Fremden spiegelt so auch die historisch-interkulturelle Spielfunktion in Rhonabwys Traum: So wie in unserer Gegenwart das Strategiespiel das >Keltische< in die Gesellschaft integriert, so wird im Gwyddbwyll das neue kontinentale Erzählen mit dem autochthonen Sagengut vermittelt.

Für das System > Spiel< kann die Überkreuzung von systematischer und diachroner Interkulturalität also gelingen. Beim Traum jedoch stößt - so scheint es - die Vermittelbarkeit an ihre Grenzen. Der Hauptgrund dafür ist, dass die moderne, freudianische Lesart des Traums die Vermittlung eines Extern-Fremden nicht mehr vorsieht. Betrachtet als Ausdruck einer Tiefenschicht der subjektiven Psyche, beraubt man den Traum der Möglichkeit, ein vermittelnder Dritter Raum zu sein. Denn hier kann das Fremde nur noch als Spiegelung des Eigenen auftauchen, als Angst beispielsweise, die ein Individuum vor dem kul-

10 | Leider erscheinen die Beiträge dieser Tagung nicht in Buchform. Ich danke aber umso herzlicher Franziska Bock und Jan Niehues für die Hinweise unter anderem auf das hier angeführte Spiel. 
turell Fremden hat - was aber nichts über das Gefürchtete, sondern lediglich etwas über die/den Fürchtenden aussagt. In der literarischen Simulation ist dies nicht anders: Wenn das kulturell Fremde auftaucht (wie - um nur eines von vielen möglichen Beispielen zu nennen - das Orientalische, das durch den Titel von Hofmannsthals Märchen der 672. Nacht angesprochen wird; vgl. Hofmannsthal 1993), so dient es keiner Vermittlung von Eigenem und Fremden. Das Fremde wird vielmehr der freudianischen Ich-Hierarchisierung unterworfen. Hofmannsthals Text hat keine interkulturelle Vermittlung des Orientalischen mit der Kultur des Fin de Siècle zum Ziel; die Bilder des Fremden sind Ausdrücke einer in sich selbst zerrissenen Psyche. Ähnliches ließe sich für viele freudianisch geprägte Traum-Texte sagen. Auch literarisch wird das Ego verabsolutiert und alles Fremde diesem untergeordnet bzw. zum Symbol des verstörten Eigenen funktionalisiert. Eine systematische Geschlossenheit, die in Rhonabwys Traum eine Vergleichbarkeit von Traum- und Spielebene gewährleistete, ist nicht mehr möglich, da das Ego und nicht der Traum die Grenzen zieht.

Die Funktionen, die der Traum in mittelalterlicher Literatur haben konnte, beispielsweise die prophetische (vgl. dazu Well 2016), gehen spätestens ab der Moderne im freudianischen Ego-Totalitarismus auf oder werden von ihm gänzlich überlagert. Genauso, wie der Glaube an eine höhere Macht die Möglichkeiten von inspirativ vermittelnden, zukunftsweisenden Traumwahrheiten bedingt, bedingt sie auch den Freiraum des Traums, als Dritter Raum zu fungieren, in dem kulturell Fremdem unhierarchisch begegnet werden kann.

Angesichts des theologisch obdachlosen und stattdessen dem Ego unterworfenen Traums der Moderne muss die Spiegelung in die Diachronie scheitern. Dieses Scheitern jedoch ist ein entscheidendes Signum der Alterität, welche als Erfolg verbucht werden kann, solange sie eben nicht als Apriori, sondern als Ergebnis des interkulturellen Vergleichs erscheint.

\section{LITERATUR}

Batts, Michael S. (1993): Der Beitrag des Mediävistikstudiums zur Interkulturalität in einer multikulturellen Gesellschaft. In: Bernd Thum/Gonthier-Louis Fink (Hg.): Praxis interkultureller Germanistik. Forschung - Bildung - Politik. Beiträge zum II. Internationalen Kongreß der Gesellschaft für Interkulturelle Germanistik. Straßburg 1991. München, S. 503-508.

Benz, Maximilian (2013): Gesicht und Schrift. Die Erzählung von Jenseitsreisen in Antike und Mittelalter. Berlin/Boston.

Ders./Weitbrecht, Julia (2011): Die Formierung des Jenseits als Bewegungsraum in Jenseitsreisen der Spätantike und des Mittelalters. In: Mittellateinisches Jahrbuch 46, H. 2, S. 229-243.

Bhabha, Homi K. (1994): The Location of Culture. London/ New York.

Birkhan, Helmut (1989): Einführung. Erläuterungen zu den einzelnen Stücken: Rhonabwys Traum. In: Ders. (Hg.; Übers.): Keltische Erzählungen vom Kaiser Arthur. Kettwig, Teil 1, S. 9-62; Teil 2, S. 201-210. 
Breulmann, Julia (2009): Erzählstruktur und Hofkultur. Weibliches Agieren in den europäischen Iweinstoff-Bearbeitungen des 12. bis 14. Jahrhunderts. Münster u.a.

Däumer, Matthias (2015) : rarcana verba quae non licet homini loqui،. Überlegungen zu Zeugenschaft und Tabu anhand der Paulusapokalypse. In: Matthias Emrich/ Alexander Dingeldein (Hg.): Texte und Tabu. Interdisziplinäre Beiträge zur Kultur von Verbot und Übertretung. Bielefeld, S. 145-171.

Ders. (2016a) "[Er] bezeugte gegen sie alle - und schrieb das Ganze». Mediale Implikationen visionärer Zeugenschaft am Beispiel der Henochapokalypse. In: Ders./Aurélia Kalisky/Heike Schlie (Hg.): ÜberZeugen. Szenarien der Zeugenschaft und ihre Akteure. München, S. 49-68.

Ders. (2016b): [Art.] "Vision / Visionsliteratur". In: Daniel Weidner (Hg.): Handbuch Religion und Literatur. Stuttgart/Weimar, S. 463-467.

Die Geschichte der Gräfin vom Brunnen [Owein or chewedyl iarlles y ffynnawn] (1989). In: Helmut Birkhan (Hg.; Übers.): Keltische Erzählungen vom Kaiser Arthur. Teil 1. Kettwig, S. 65-107.

Görling, Reihnhold (1999): A Hot Thing. Über die Nähe des Anderen. In: Stefan Rieger/ Schamma Schahadat/Manfred Weinberg (Hg.): Interkulturalität. Zwischen Inszenierung und Archiv. Tübingen, S. 269-284.

Herweg, Mathias (2017): Alterität und Kontinuität. Vom interkulturellen Potential der germanistischen Mediävistik. In: Zeitschrift für interkulturelle Germanistik 8, H. 1, S. 11-23.

Hofmannsthal, Hugo von (1993): Das Märchen der 672. Nacht. In: Ders.: Gesammelte Werke in zehn Einzelbänden. Erzählungen, Erfundene Gespräche und Briefe, Reisen. Hg. v. Bernd Schoeller. Frankfurt a.M., S. 45-66.

Le Goff, Jaques (1990): Die Geburt des Fegefeuers. Vom Wandel des Weltbilds im Mittelalter. Übers. v. Ariane Forkel. München.

Mersey, Daniel (2011): Song of Arthur and Merlin. Arthurian Skirmish Miniatures Rules. Narni.

Müller, Ulrich (1993): Mediävistik und interkulturelle Germanistik: Praxisbeispiele einer sinnvollen Symbiose. In: Bernd Thum/Gonthier-Louis Fink (Hg.): Praxis interkultureller Germanistik. Forschung - Bildung - Politik. Beiträge zum II. Internationalen Kongreß der Gesellschaft für Interkulturelle Germanistik, Straßburg 1991. München, S. 855-867.

Niehues, Jan (2013): Die Brettspiele des mittelalterlichen Irland und Wales. In: Matthias Teichert (Hg.): Sport und Spiel bei den Germanen. Nordeuropa von der römischen Kaiserzeit bis zum Mittelalter. Berlin, S. 217-244.

Rhonabwys Traum [Breuddwyd Rhonabwy] (1989). In: Helmut Birkhan (Hg.; Übers.): Keltische Erzählungen vom Kaiser Arthur. Teil 2. Kettwig, S. 119-142.

Shitanda, So (1993): Eine kognitiv-psychologische Analyse zum Image des europäischen Mittelalters und der deutschen Gegenwart in Japan. In: Bernd Thum/Gonthier-Louis Fink (Hg.): Praxis interkultureller Germanistik. Forschung - Bildung - Politik. Beiträge zum II. Internationalen Kongreß der Gesellschaft für Interkulturelle Germanistik, Straßburg 1991. München, S. 713-732.

Weitbrecht, Julia (2011): Aus der Welt. Reise und Heilung in Legenden und Jenseitsreisen der Spätantike und des Mittelalters. Heidelberg. 
Well, Benjamin van (2016): Mir troumt hînaht ein troum. Untersuchung zur Erzählweise von Träumen in mittelhochdeutscher Epik. Göttingen.

Zimmermann, Peter (Hg.; $\left.{ }^{2} 1991\right)$ : Interkulturelle Germanistik. Dialog der Kulturen auf Deutsch? Frankfurt a.M. 
